\title{
Stability analysis of yield and capsaicinoids content in chili (Capsicum spp.) grown across six environments
}

\author{
Tulsi Gurung • Suchila Techawongstien • \\ Bhalang Suriharn $\cdot$ Sungcom Techawongstien
}

Received: 31 October 2011/ Accepted: 16 March 2012/Published online: 1 April 2012

(C) The Author(s) 2012. This article is published with open access at Springerlink.com

\begin{abstract}
There is a need for identifying chili cultivars with stable amounts of capsaicinoids so that the amount of pungency in the final product can be controlled. Therefore, six cultivars were evaluated for yield and capsaicinoid contents across six environments, four in Thailand and two in Bhutan, ranging from 200 to 1,630 $\mathrm{m}$ above mean sea level. Combined analyses showed significant differences among cultivars, environments, and for cultivar by environment interactions for fruit yields, individual capsaicinoid and total capsaicinoid contents. A large proportion (46.1\%) of variation on yield was attributed to environments; however, for total capsaicinoid contents, genotype effect accounted for $74.2 \%$ of variation. Variation due to environment was $5.8 \%$, while for cultivar by environment interaction was $15.8 \%$. Cultivar Dallay khorsaney had high capsaicin, dihydrocapsaicin and total capsaicinoids but was very sensitive to environmental changes, and therefore good for specific adaptation. Cultivar KKU-P-11003 with total capsaicinoid contents of 78,721 Scoville
\end{abstract}

T. Gurung

College of Natural Resources, Royal University

of Bhutan, Lobesa, Punakha, Bhutan

S. Techawongstien $(\bowtie) \cdot$ B. Suriharn .

S. Techawongstien

Department of Plant Science and Agricultural Resources,

Faculty of Agriculture, Khon Kaen University,

Khon Kaen 40002, Thailand

e-mail: suctec.kku@gmail.com heat unit was stable for dry fruit yield, capsaicin, dihydrocapsaicin, and total capsaicinoids with regression coefficients $b=1.06,1.06,0.78$, and 0.96 respectively. Therefore, KKU-P-11003 was considered suitable for diverse environments. In addition, this result indicates that it is possible to select stable cultivar for capsaicinoid contents.

Keywords Capsaicin - Cultivar - Dihydrocapsaicin . Environment · Genotype · Interaction

\section{Introduction}

Stability of pungency in fresh chili and in processed chili products is one of the concerns in food industries. The manufacturers can label the food products as hot, medium or low if the specific pungency levels are maintained (Zewdie and Bosland 2000). Moreover, capsaicinoids use in pharmaceutical and cosmetic industries is increasing. Each of these applications requires a specific level of capsaicinoids. Therefore, there is a need for identifying chili cultivars with stable amounts of capsaicinoids so the amount of pungency in the final product can be controlled. A genotype or cultivar that shows consistent performance across different environments and years for a given trait is considered stable. Although, the pungency characteristic is affected by genotype and environmental factors (Zewdie and Bosland 2000), cultivar has a major effect (Gurung et al. 2011). Therefore, it is possible to select 
widely adapted stable cultivar for pungency. Plant breeders can selectively develop cultivars with certain ranges of pungency. Partitioning of growing environments to reduce genotype $\times$ environment $(G \times E)$ interaction is challenging especially in regions where climatic variation is large. Therefore, evaluation of cultivars by stability parameters across multi-environments is important to identify the consistent performing and high yielding cultivars.

There are several methods developed to assess stability of cultivars across environments. However, each method has its advantages and limitations. Combined analysis of variance (ANOVA) has been used to detect $G \times$ E interactions and their magnitude. However, this analysis does not provide the measurement of response by individual genotype to environments. Regression technique was proposed by Finlay and Wilkinson (1963) and was improved by Eberhart and Russell (1966). This is a popular method in stability analysis and has been applied in many crops. Given the limitation of information on the stability of capsaicinoid contents in chili, this study was conducted across six environments to understand the responses and to identify varietal stability on capsaicinoids.

\section{Materials and methods}

Plant materials and field experiments

Six cultivars (Table 1) from different sources and with different pungency levels were used for stability test. These cultivars were sampled among the most promising cultivars from the previous experiments. Field experiments were conducted across six environments, four in Thailand and two in Bhutan. In Thailand, the main growing season or rainy season experiments were conducted from June-October 2009 and dry season was done from October 2010-April 2011 at Khon Kaen (KK) University farm $\left(18^{\circ} 51^{\prime} \mathrm{N}\right.$ and $98^{\circ} 45^{\prime} \mathrm{E}$, at $200 \mathrm{~m}$ a.s.l) and at Royal Project Foundation Research Centre Pangda, Chiang Mai (CM) $\left(16^{\circ} 28^{\prime} \mathrm{N}\right.$ and $102^{\circ} 48^{\prime} \mathrm{E}$, at $680 \mathrm{~m}$ a.s.l). In Bhutan, experiments were conducted during chili growing season i.e. April-September 2010 at the College of Natural Resources farm, Lobesa (LB) $\left(27^{\circ} 30^{\prime} \mathrm{N}\right.$ and $89^{\circ} 52^{\prime} \mathrm{E}$, at $1,400 \mathrm{~m}$ a.s.l) and a farmer's field in the Kabesa sub-district $(\mathrm{KB}),\left(27^{\circ} 38^{\prime} \mathrm{N}\right.$ and $89^{\circ} 52^{\prime} \mathrm{E}$, at $1,630 \mathrm{~m}$ a.s.l). A randomized complete block design with three replications was used in all experiments. The plants were spaced $60 \mathrm{~cm}$ between plants, and $50 \mathrm{~cm}$ between rows. Standard crop management practices, through nursery to harvest, were followed in all locations. Drip irrigation system was laid out in all the experiments so that soil moisture was not limiting. Environment data such as soil properties, temperature, relative humidity, rainfall and solar radiation were recorded (Table 2). Four subsequent harvests were done and fruits were sundried for 2 days and then oven dried at $80{ }^{\circ} \mathrm{C}$ for $12-24 \mathrm{~h}$ to obtain the constant dry weight.

Capsaicinoids analysis

Capsaicinoids were extracted and quantified according to the 'short run' method with high performance liquid chromatography (HPLC), and the data was converted to Scoville heat unit (SHU), as described by Collins et al. (1995).

Table 1 Descriptions of chili cultivars used in experiments

\begin{tabular}{|c|c|c|c|c|c|c|}
\hline Cultivar & Pedigree name & Source & Fruit type & Pungency $^{\mathrm{a}}$ & Species & Fruit characteristics \\
\hline KKU-P-11015 & Num keaw tong 80 & Thailand & Long cayenne & Low & C. аппиит & Medium, elongated, pointed end \\
\hline KKU-P-11175 & - & Thailand & Long cayenne & Low & C. аппиит & Large, elongated, pointed end \\
\hline KKU-P-11003 & Yodson khem 80 & Thailand & Chili & Medium & C. аппиит & Small, elongated, pointed end \\
\hline Dallay Khorsaney & - & Bhutan & Chili & High & C. chinense & Small, round to oblong \\
\hline KKU-P-21041 & $\begin{array}{l}\text { C05680-1 } \\
\quad \text { (Punjab Lal) }\end{array}$ & India & Chili & High & C. аппиит & Small, elongated, pointed end \\
\hline KKU-P-22006 & $\mathrm{C} 00307$ & Taiwan & Bird chili & High & C. аппиит & Small, elongated, pointed end \\
\hline
\end{tabular}

${ }^{a}<50,000$ SHU is considered as low pungency; 50,000-100,000 SHU as medium pungency; $>100,000$ SHU as high pungency 
Table 2 Descriptions of environments where trials were conducted during 2009-2011

\begin{tabular}{|c|c|c|c|c|c|c|c|c|c|c|}
\hline \multirow[t]{2}{*}{ Environments } & \multirow[t]{2}{*}{ Planting date } & \multirow[t]{2}{*}{$\begin{array}{l}\text { Geographical } \\
\text { coordinates }\end{array}$} & \multirow[t]{2}{*}{$\begin{array}{l}\text { Altitude } \\
\text { (m asl) }\end{array}$} & \multicolumn{2}{|c|}{$\begin{array}{l}\text { Temperature } \\
\left({ }^{\circ} \mathrm{C}\right)\end{array}$} & \multicolumn{2}{|c|}{$\begin{array}{l}\text { Relative } \\
\text { Humidity } \\
(\%)\end{array}$} & \multirow[t]{2}{*}{$\begin{array}{l}\text { Rainfall } \\
(\mathrm{mm})\end{array}$} & \multirow[t]{2}{*}{$\begin{array}{l}\text { Solar radiation } \\
\left(\mathrm{MJ} \mathrm{m}^{-2} \mathrm{day}^{-1}\right)\end{array}$} & \multirow[t]{2}{*}{ Soil type } \\
\hline & & & & $\operatorname{Max}$ & Min & $\operatorname{Max}$ & Min & & & \\
\hline $\begin{array}{l}\text { Khon Kaen } \\
\text { (KK1) }\end{array}$ & $\begin{array}{l}\text { June } \\
2009\end{array}$ & $\begin{array}{l}16^{\circ} 28^{\prime} \mathrm{N} \\
102^{\circ} 48^{\prime} \mathrm{E}\end{array}$ & 200 & 34.8 & 23.6 & 88.4 & 61.7 & 133.3 & 23.7 & $\begin{array}{l}\text { Sandy } \\
\text { loam }\end{array}$ \\
\hline $\begin{array}{l}\text { Chiang Mai } \\
\text { (CM1) }\end{array}$ & $\begin{array}{l}\text { June } \\
2009\end{array}$ & $\begin{array}{l}18^{\circ} 51^{\prime} \mathrm{N} \\
98^{\circ} 45^{\prime} \mathrm{E}\end{array}$ & 680 & 30.4 & 22.1 & 85.4 & 60.8 & 219.6 & 20.6 & Red clay \\
\hline Lobesa (LB) & $\begin{array}{l}\text { April } \\
2010\end{array}$ & $\begin{array}{l}27^{\circ} 30^{\prime} \mathrm{N} \\
89^{\circ} 52^{\prime} \mathrm{E}\end{array}$ & 1,400 & 32.2 & 16.4 & 92.1 & 55.0 & 103.2 & 18.1 & $\begin{array}{c}\text { Sandy } \\
\text { clay } \\
\text { loam }\end{array}$ \\
\hline Kabesa (KB) & $\begin{array}{l}\text { April } \\
2010\end{array}$ & $\begin{array}{l}27^{\circ} 38^{\prime} \mathrm{N} \\
89^{\circ} 52^{\prime} \mathrm{E}\end{array}$ & 1,630 & 30.5 & 13.6 & 88.0 & 52.0 & 206.1 & 18.9 & $\begin{array}{c}\text { Sandy } \\
\text { clay } \\
\text { loam }\end{array}$ \\
\hline $\begin{array}{l}\text { Khon Kaen } \\
\text { (KK2) }\end{array}$ & $\begin{array}{c}\text { October } \\
2010\end{array}$ & $\begin{array}{l}16^{\circ} 28^{\prime} \mathrm{N} \\
102^{\circ} 48^{\prime} \mathrm{E}\end{array}$ & 200 & 31.3 & 19.5 & 83.1 & 56.3 & 0.8 & 10.3 & Loamy sand \\
\hline $\begin{array}{l}\text { Chiang Mai } \\
\text { (CM2) }\end{array}$ & $\begin{array}{c}\text { October } \\
2010\end{array}$ & $\begin{array}{l}18^{\circ} 51^{\prime} \mathrm{N} \\
98^{\circ} 45^{\prime} \mathrm{E}\end{array}$ & 680 & 28.0 & 17.6 & 88.6 & 54.5 & 1.1 & 16.4 & Silty loam \\
\hline
\end{tabular}

Data analysis

Yield and capsaicinoid traits were statistically analyzed for each environment. Error variances were tested for homogeneity with Bartlett's test as described by Gomez and Gomez (1984). Duncan multiple range test (DMRT) was used to calculate to compare mean differences for significant cultivar and environment effect by using MSTAT-C software (Russel 1994). Combined analysis of variance was done for six environments according to a statistical model explained by Freeman and Dowker (1973). Since there was significant interactions between $\mathrm{G} \times \mathrm{E}$, stability parameters were calculated as suggested by Eberhart and Russell (1966). Means across environments, linear regression coefficient $(b)$, deviation from regression $\left(\mathrm{Sd}^{2}\right)$ of genotypes means over environment index were calculated. Regression coefficient $(b)$ value was tested for its difference from 0 and if significant it was tested from 1.

\section{Results}

Cultivar by environment interaction

The results of combined analysis of variance for yield and capsaicinoid traits are presented in Table 3 . There were significant differences among cultivars, environments, and for cultivar by environment interactions for all traits. A large proportion (47.9\%) of variation on yield in dry weight per plant was attributed to environments. Source of variation on yield by Cultivar (G) $\times$ environment (E) and cultivar accounted, respectively, for 19.5 and $19.5 \%$ of the total variation. However, for capsaicinoids high variations due to cultivar were observed for individual and total capsaicinoid contents. Variations due to cultivar were 71.9, 68.7, and $74.2 \%$ for capsaicin, dihydrocapsaicin, and total capsaicinoid contents, respectively.

\section{Environment evaluation}

Due to highly significant differences among cultivar by environment interactions, the mean of six cultivars for yield and capsaicinoid traits from each environment was used to rank the environmental effects on each trait as suggested by Finlay and Wilkinson (1963). Among varieties, KKU-P-11015 produced high mean yield of $174.8 \mathrm{~g} /$ plant across six environments, however, it was not significantly different from KKU-11175, Dallay khorsaney and KKU-P-21041 (Table 4). KK1 in rainy season was the most favorable environment with mean yield of $196 \mathrm{~g} /$ plant. Mean yield at Kabesa Bhutan, during rainy season was not 
Table 3 Combined analysis of variance for yield and capsaicinoid traits of six chili cultivars evaluated in six environments during 2009-2011

\begin{tabular}{|c|c|c|c|c|c|}
\hline \multirow[t]{2}{*}{ Source of variation } & \multirow[t]{2}{*}{ df } & \multicolumn{4}{|l|}{ Mean Square } \\
\hline & & Dry weight (g/plant) & Capsaicin (SHU) & Dihydrocapsaicin (SHU) & Total capsaicinoid (SHU) \\
\hline Cultivars (G) & 5 & $12,966.2(19.5)^{* *}$ & $2.77 \times 10^{10}(71.9)^{* *}$ & $1.19 \times 10^{10}(68.7)^{* *}$ & $7.56 \times 10^{10}(74.2)^{* *}$ \\
\hline Environment (E) & 5 & $31797.5(47.9)^{* *}$ & $3.32 \times 10^{9}(8.6)^{* *}$ & $1.21 \times 10^{9}(7.0)^{* *}$ & $5.92 \times 10^{9}(5.8)^{* *}$ \\
\hline Error $(E \times \operatorname{Rep})^{\mathrm{a}}$ & 5 & $810.0(2.9)$ & $1.07 \times 10^{8}(0.7)$ & $6.84 \times 10^{7}(0.9)$ & $3.1 \times 10^{8}(0.7)$ \\
\hline $\mathrm{G} \times \mathrm{E}$ & 12 & $2576.4(19.5)^{* *}$ & $1.15 \times 10^{9}(14.9)^{* *}$ & $\begin{array}{l}6.81 \times 10^{8} \\
(19.7)^{* *}\end{array}$ & $3.21 \times 10^{9}(15.8)^{* *}$ \\
\hline Error $(E \times \operatorname{Rep} \times G)^{b}$ & 25 & $563.4(10.2)$ & $1.24 \times 10^{8}(3.9)$ & $5.29 \times 10^{7}(3.7)$ & $2.97 \times 10^{8}(3.5)$ \\
\hline $\mathrm{CV}(\%)^{\mathrm{a}}$ & & 19.4 & 17.0 & 21.4 & 17.9 \\
\hline $\mathrm{CV}(\%)^{\mathrm{b}}$ & & 16.2 & 18.2 & 18.9 & 17.3 \\
\hline
\end{tabular}

Numbers in parentheses are $\%$ sum of squares which shows the $\%$ of variation

${ }^{\mathrm{a}}$ and ${ }^{\mathrm{b}}$ shows coefficient of variation due to error $(\mathrm{E} \times \operatorname{Rep})$ and error $(\mathrm{E} \times \operatorname{Rep} \times \mathrm{G})$, respectively

** Significant at $P \leq 0.01$ probability level

significantly different from yield during dry season at Khon Kaen and Chaing Mai. The variation on individual yield ranged from $44.4 \mathrm{~g} /$ plant for KKUP-11003 at Chiang Mai dry season to 270.7 g/plant for KKU-P-11015 at Khon Kaen during rainy season.

Capsaicin, dihydrocapsaicin, and total capsaicinoids showed similar responses among cultivars across six environments. Dallay khorsaney produced the highest capsaicin (115532.5 SHU), dihydrocapsaicin (74212.1 SHU), and total capsaicinoids (187100.7
SHU) among cultivars, across all environments (Table 5). Environmental mean for capsaicin $(79,048$ $\mathrm{SHU}$ ) was highest at Kabesa during rainy season. For dihydrocapsaicin (46257.3 SHU) it was highest at Khon Kaen during dry season. For total capsaicinoids the highest value (120,394 SHU) was obtained at Chiang Mai during rainy season. In addition, among individual cultivars, Dallay khorsaney produced the maximum capsaicin (160471.3 SHU) at Chaing Mai during rainy season. However, dihydrocapsaicin

Table 4 Dry fruit weight (g/plant) of six chili cultivars grown at six environments during 2009-2011

\begin{tabular}{|c|c|c|c|c|c|c|c|}
\hline \multirow[b]{2}{*}{ Cultivars (G) } & \multicolumn{7}{|c|}{ Environments (E) } \\
\hline & KK1 & CM1 & LB & $\mathrm{KB}$ & KK2 & $\mathrm{CM} 2$ & Mean \\
\hline KKU-P-11015 & $270.7^{\mathrm{a}}$ & 170.0 & 221.3 & $136.6^{\mathrm{b}}$ & $130.3^{\mathrm{a}}$ & $119.5^{b}$ & $174.8^{\mathrm{A}}$ \\
\hline KKU-P-11175 & $192.7^{\mathrm{b}}$ & 191.3 & 207.1 & $126.2^{b c}$ & $142.4^{\mathrm{a}}$ & $153.0^{\mathrm{a}}$ & $168.8^{\mathrm{A}}$ \\
\hline KKU-P-11003 & $173.0^{\mathrm{b}}$ & 132.3 & 157.7 & $72.2^{c}$ & $91.9^{b}$ & $44.4^{\mathrm{d}}$ & $112.0^{\mathrm{C}}$ \\
\hline Dallay khorsaney & $177.5^{b}$ & 195.2 & 218.7 & $198.8^{\mathrm{a}}$ & $86.1^{\mathrm{c}}$ & $71.2^{\mathrm{cd}}$ & $158.6^{\mathrm{AB}}$ \\
\hline KKU-P-21041 & $210.8^{b}$ & 191.8 & 152.7 & $109.3^{b c}$ & $93.6^{b}$ & $137.1^{\mathrm{ab}}$ & $149.3^{\mathrm{AB}}$ \\
\hline KKU-P-22006 & $153.0^{\mathrm{b}}$ & 188.4 & 138.3 & $70.4^{\mathrm{c}}$ & $92.9^{b}$ & $81.2^{\mathrm{c}}$ & $120.7^{\mathrm{BC}}$ \\
\hline Mean & $196.0^{\mathrm{A}}$ & $178.2^{\mathrm{A}}$ & $182^{\mathrm{A}}$ & $119.0^{\mathrm{B}}$ & $106.3^{\mathrm{B}}$ & $101.1^{\mathrm{B}}$ & 147.4 \\
\hline$F$ test & $* *$ & $\mathrm{~ns}$ & $\mathrm{~ns}$ & $* *$ & $* *$ & $* *$ & \\
\hline $\mathrm{CV} \%$ & 10.4 & 16.3 & 20.4 & 18.4 & 13.8 & 11.8 & \\
\hline
\end{tabular}

KK1 Khon Kaen rainy season 2009, CM1 Chiang Mai rainy season 2009, LB Lobesa rainy season 2010, KB Kabesa rainy season 2010, KK2 Khon Kaen dry season 2010-2011, CM2 Chiang Mai dry season 2010-2011

** Significant at $P \leq 0.01$ probability level. Mean in the same column and row followed by a common letter are not significantly different at $P \leq 0.01$ by DMRT. Different capital letter(s) indicate significant difference between environments and between cultivars 
(137,077 SHU) and total capsaicinoids (266,405 SHU) during dry season was highest at Khon Kaen. In summary, all capsaicinoid traits among cultivars followed this ranking: Dallay khorsaney $>$ KKU-P$21041>$ KKU-P-22006 > KKU-P-11003 > KKUP-11015 > KKU-P-11175 (Table 5). However, there was no significant difference between KKU-P-21041 and KKU-P-22006.
Stability for yield and capsaicinoids

Stability parameters for yield and capsaicinoid traits are shown in Table 6. KKU-P-11003 showed good stability for all traits with $b=1.06,1.06,0.78$, and 0.96 for dry fruit yield, capsaicin, dihydrocapsaicin, and total capsaicinoids respectively (Table 6). It indicates general adaptability in all traits. KKU-P-

Table 5 Capsaicin, dihydrocapsaicin and total capsaicinoid contents (SHU) of six chili cultivars grown at six environments during 2009-2011

\begin{tabular}{|c|c|c|c|c|c|c|c|}
\hline \multirow[t]{2}{*}{ Cultivars (G) } & \multicolumn{6}{|c|}{ Environments (E) } & \multirow[b]{2}{*}{ Mean } \\
\hline & KK1 & CM1 & LB & $\mathrm{KB}$ & $\mathrm{KK} 2$ & CM2 & \\
\hline \multicolumn{8}{|c|}{ Capsaicin contents (SHU) } \\
\hline KKU-P-11015 & $21794.4^{\mathrm{cd}}$ & $26201.1^{\mathrm{d}}$ & $20828.0^{\mathrm{cd}}$ & $30992.4^{\mathrm{d}}$ & $25401.0^{\mathrm{d}}$ & $7249.4^{c}$ & $22077.7^{\mathrm{D}}$ \\
\hline KKU-P-11175 & $20565.9^{d}$ & $8043.7^{\mathrm{d}}$ & $3752.2^{\mathrm{d}}$ & $38397.2^{\mathrm{cd}}$ & $8011.3^{\mathrm{e}}$ & $5513.4^{\mathrm{c}}$ & $14047.3^{\mathrm{E}}$ \\
\hline KKU-P-11003 & $35001.5^{\mathrm{cd}}$ & $63727.5^{\mathrm{c}}$ & $41159.9^{\mathrm{bc}}$ & $72290.4^{\mathrm{bc}}$ & $52297.6^{\mathrm{c}}$ & $40514.7^{\mathrm{b}}$ & $50831.9^{\mathrm{C}}$ \\
\hline Dallay khorsaney & $104688.8^{\mathrm{a}}$ & $160471.3^{\mathrm{a}}$ & $108945.6^{\mathrm{a}}$ & $108356.7^{\mathrm{a}}$ & $145198.5^{\mathrm{a}}$ & $65535.7^{\mathrm{b}}$ & $115532.8^{\mathrm{A}}$ \\
\hline KKU-P-21041 & $40958.7^{c}$ & $93429.6^{\mathrm{bc}}$ & $60974.1^{\mathrm{b}}$ & $131392.2^{\mathrm{a}}$ & $73858.1^{\mathrm{b}}$ & $102209.9^{\mathrm{a}}$ & $83803.7^{\text {B }}$ \\
\hline KKU-P-22006 & $69201.5^{\mathrm{b}}$ & $106572.3^{b}$ & $60707.9^{b}$ & $92865.0^{\mathrm{ab}}$ & $57941.9^{\mathrm{c}}$ & $94226.4^{\mathrm{a}}$ & $80252.5^{\mathrm{B}}$ \\
\hline Mean & $48701.8^{\mathrm{CD}}$ & $76407.6^{\mathrm{A}}$ & $49394.6^{\mathrm{D}}$ & $79049.0^{\mathrm{A}}$ & $60451.4^{\mathrm{AB}}$ & $52541.6^{\mathrm{BC}}$ & 61091.0 \\
\hline$F$ test & $* *$ & $* *$ & $* *$ & $* *$ & $* *$ & $* *$ & \\
\hline $\mathrm{CV} \%$ & 16.1 & 18.5 & 20.0 & 20.0 & 7.5 & 20.2 & \\
\hline \multicolumn{8}{|c|}{ Dihydrocapsaicin contents (SHU) } \\
\hline KKU-P-11015 & $17207.4^{\mathrm{d}}$ & $15175.0^{\mathrm{bc}}$ & $12246.8^{c}$ & $15600.5^{\mathrm{b}}$ & $17877.6^{\text {de }}$ & $10983.9^{\mathrm{d}}$ & $14848.5^{\mathrm{D}}$ \\
\hline KKU-P-11175 & $11072.0^{\mathrm{d}}$ & $2410.0^{c}$ & $2379.5^{\mathrm{bc}}$ & $19415.5^{\mathrm{b}}$ & $4963.6^{\mathrm{e}}$ & $679.6^{\mathrm{d}}$ & $6820.0^{\mathrm{E}}$ \\
\hline KKU-P-11003 & $29755.8^{c}$ & $34194.7^{\mathrm{b}}$ & $14689.9^{\mathrm{bc}}$ & $27302.3^{b}$ & $25234.9^{\mathrm{cd}}$ & $31414.7^{\mathrm{c}}$ & $27098.7^{\mathrm{C}}$ \\
\hline Dallay khorsaney & $76476.6^{\mathrm{a}}$ & $79458.3^{\mathrm{a}}$ & $49429.1^{\mathrm{a}}$ & $57787.7^{\mathrm{a}}$ & $137077.4^{\mathrm{a}}$ & $45043.5^{b}$ & $74212.1^{\mathrm{A}}$ \\
\hline KKU-P-21041 & $38768.2^{\mathrm{c}}$ & $63170.9^{\mathrm{a}}$ & $34376.9^{\mathrm{a}}$ & $52321.0^{\mathrm{a}}$ & $52493.4^{\mathrm{b}}$ & $86500.6^{a}$ & $54605.1^{\text {В }}$ \\
\hline KKU-P-22006 & $53189.5^{b}$ & $69506.9^{\mathrm{a}}$ & $30972.9^{\mathrm{ab}}$ & $46820.4^{\mathrm{a}}$ & $39896.5^{b c}$ & $76768.6^{\mathrm{a}}$ & $52859.1^{\text {В }}$ \\
\hline Mean & $37744.9^{\mathrm{B}}$ & $43985.9^{\mathrm{AB}}$ & $24015.9^{\mathrm{C}}$ & $36541.2^{\mathrm{B}}$ & $46257.2^{\mathrm{A}}$ & $41898.5^{\mathrm{AB}}$ & 8407.3 \\
\hline$F$ test & $* *$ & ** & $* *$ & $* *$ & $* *$ & $* *$ & \\
\hline $\mathrm{CV} \%$ & 12.2 & 24.0 & 31.4 & 19.1 & 15.3 & 12.4 & \\
\hline \multicolumn{8}{|c|}{ Total capsaicinoid contents (SHU) } \\
\hline KKU-P-11015 & $39001.8^{\mathrm{de}}$ & $41376.1^{\mathrm{de}}$ & $33075.8^{\mathrm{cd}}$ & $46593.9^{\mathrm{c}}$ & $45566.1^{\mathrm{bc}}$ & $18106.2^{\mathrm{d}}$ & $37286.3^{\mathrm{D}}$ \\
\hline KKU-P-11175 & $31637.9^{e}$ & $10454.7^{\mathrm{e}}$ & $6132.6^{\mathrm{d}}$ & $57813.8^{\mathrm{c}}$ & $12975.3^{\mathrm{c}}$ & $6193.0^{\mathrm{d}}$ & $20867.4^{\mathrm{E}}$ \\
\hline KKU-P-11003 & $64757.3^{\mathrm{cd}}$ & $97922.2^{\mathrm{cd}}$ & $55850.8^{\mathrm{bc}}$ & $99593.6^{b}$ & $82276.4^{b}$ & $71929.4^{\mathrm{c}}$ & $78721.3^{\mathrm{C}}$ \\
\hline Dallay khorsaney & $181165.4^{\mathrm{a}}$ & $239930.6^{\mathrm{a}}$ & $158375.7^{\mathrm{a}}$ & $166144.4^{\mathrm{a}}$ & $266405.0^{\mathrm{a}}$ & $110579.2^{b}$ & $187100.7^{\mathrm{A}}$ \\
\hline KKU-P-21041 & $79727.0^{\mathrm{c}}$ & $15660.5^{\mathrm{c}}$ & $9535.0^{\mathrm{b}}$ & $183713.1^{\mathrm{a}}$ & $136836.6^{b}$ & $188711.5^{\mathrm{a}}$ & $140156.3^{\mathrm{B}}$ \\
\hline KKU-P-22006 & $12239.9^{b}$ & $176079.2^{b}$ & $9168.8^{\mathrm{b}}$ & $139685.4^{\mathrm{a}}$ & $103802.9^{b}$ & $170995.9^{\mathrm{a}}$ & $134106.5^{\mathrm{B}}$ \\
\hline Mean & $86447.0^{\mathrm{CD}}$ & $120394.0^{\mathrm{A}}$ & $73410.0^{\mathrm{D}}$ & $115590.0^{\mathrm{A}}$ & $107977.0^{\mathrm{AB}}$ & $94419.0^{\mathrm{BC}}$ & 99705.5 \\
\hline$F$ test & $* *$ & $* *$ & ** & $* *$ & $* *$ & ** & \\
\hline $\mathrm{CV} \%$ & 13.9 & 19.2 & 24.1 & 23.6 & 8.0 & 15.6 & \\
\hline
\end{tabular}

KK1 Khon Kaen rainy season 2009, CM1 Chiang Mai rainy season 2009, LB Lobesa rainy season 2010, KB Kabesa rainy season 2010, KK2 Khon Kaen dry season 2010-2011, CM2 Chiang Mai dry season 2010-2011

** Significant at $P \leq 0.01$ probability level. Mean in the same column followed by a common letter are not significantly different at $P \leq 0.01$ by DMRT. Different capital letter(s) indicates significant difference between environments and between cultivars at $P \leq 0.01$ 
Table 6 Stability analyses for yield and capsaicinoids of six chili cultivars grown at six environments during 2009-2011

\begin{tabular}{|c|c|c|c|c|c|c|c|c|c|c|c|c|}
\hline \multirow[t]{2}{*}{ Cultivars } & \multicolumn{3}{|c|}{ Dry fruit weight (g/plant) } & \multicolumn{3}{|c|}{ Capsaicin (SHU) } & \multicolumn{3}{|c|}{ Dihydrocapsaicin (SHU) } & \multicolumn{3}{|c|}{ Total capsaicinoid (SHU) } \\
\hline & Mean & $b$ & $\mathrm{Sd}^{2}$ & Mean & $b$ & $\mathrm{Sd}^{2}$ & Mean & $b$ & $\mathrm{Sd}^{2}$ & Mean & $b$ & $\mathrm{Sd}^{2}$ \\
\hline KKU-P-11015 & 174.8 & 1.26 & 28.0 & 22,078 & $0.39^{* *}$ & 6,774 & 14,849 & $0.14^{* * *}$ & 2,750 & 37,286 & $0.30^{* *}$ & 10,176 \\
\hline KKU-P-11175 & 168.8 & 0.67 & 16.4 & 14,047 & $0.50^{* *}$ & 12,978 & 6,820 & $-0.06^{* *}$ & 7,990 & 20,867 & $0.38^{* *}$ & 21,470 \\
\hline KKU-P-11003 & 112.0 & 1.06 & 17.7 & 50,832 & 1.06 & 3,078 & 27,099 & 0.78 & 4,129 & 78,721 & 0.96 & 3,917 \\
\hline Dallay khorsaney & 158.6 & $1.08^{* *}$ & 47.0 & 115,533 & $1.28^{* *}$ & 36,455 & 74,212 & $2.28^{* * *}$ & 30,248 & 187,100 & $1.65^{* *}$ & 54,655 \\
\hline KKU-P-21041 & 149.3 & 0.91 & 26.2 & 83,804 & $1.79^{* *}$ & 23,450 & 54,605 & $1.60^{* *}$ & 16,763 & 140,156 & $1.61^{* *}$ & 37,651 \\
\hline KKU-P-22006 & 120.7 & 0.94 & 25.6 & 80,252 & $0.96^{* *}$ & 18,289 & 52,859 & $1.55^{* *}$ & 16,004 & 134,106 & $1.08^{* *}$ & 33,824 \\
\hline Mean & 147.4 & & & 61,091 & & & 38,407 & & & 99,706 & & \\
\hline
\end{tabular}

Significant from 1.0 at $P \leq 0.01$ probability level indicates unstable cultivars

21041 with regression coefficient of $b=0.91$ and with above average dry fruit yield (location mean yield $=149.3 \mathrm{~g} /$ plant) indicated general adaptability for fruit yield. Although, KKU-P-11003 and KKU-P22006 had regression coefficient for yield of $b=1.06$ and 0.94 respectively, their average yield was low, therefore more stable in diverse environments but in general low yielding. KKU-P-11015 gave high yield but had a higher regression coefficient of $b=1.26$ indicating that this cultivar performed well under favorable conditions. Dallay khorsaney produced high yield and showed stability ( $\left.b=1.08^{* *}\right)$, but significantly different from 1.0 and high deviation from regression (47.0). This showed that this cultivar is very sensitive to changes in environment.

KKU-P-11003 was the only cultivar that showed stability for capsaicinoids. All other cultivars showed $b$ value significantly different from 1.0 and high deviation from regression, indicating high fluctuation in capsaicinoid contents across environments. In KKU-P-22006, capsaicin and total capsaicinoid content were stable i.e. $b=0.96 * *$ and $1.08^{* *}$, respectively. However, it was sensitive for dihydrocapsaicin $\left(b=1.55^{* *}\right)$. Dallay khorsaney had the highest of all capsaicinoid traits, but $b>1$ and high deviation from regression. Therefore, this cultivar is considered to be good only for specific locations. KKU-P-11015 showed low coefficient of regression value for capsaicin $\left(b=0.39^{* *}\right)$ and dihydrocapsaicin $\left(b=0.14^{* *}\right)$. Similarly, KKU-P-11175 showed low regression values for capsaicin $\left(b=0.50^{* *}\right)$ and for dihydrocapsaicin $\left(-0.06^{* *}\right)$. These indicate less responsiveness to changes in environments for capsaicinoids in these two cultivars.

\section{Discussion}

Significant $G \times E$ effects indicated that cultivar responded differently to changes in environments. High proportion of variation on yield was found for the environment effect, therefore more testing sites are needed or the environments in locations need to be controlled (Gill et al. 1984). Many studies reported that capsaicinoids content is affected by genetic and environment conditions (Iwai et al. 1979; ContrerasPadilla and Yahia 1998; Estrada et al. 1999; Zewdie and Bosland 2000). Moreover, Harvell and Bosland (1997) reported environment has stronger effect on capsaicinoids. In contrast, we found that cultivar plays a major role in capsaicinoid contents as more than $70 \%$ of the variation was due to cultivar effect although $\mathrm{G} \times \mathrm{E}$ were significant. A large source of variation due to genotype was also reported by Zewdie and Bosland (2000) in their study of haploid, F1 hybrid and open pollinated genotypes, in three different environments. However, our cultivars were local varieties from several countries and were grown across six environments. Therefore, even with diverse environments, cultivars had more effect on capsaicinoids than environments.

Since there was significant cultivar by environment interaction it will lessen the usefulness of cultivar mean as single parameter to measure stability (Rasamivelona et al. 1995; Pritts and Luby 1990). Therefore, stability analyses were done. According to Eberhart and Russell (1966) model, a genotype is considered stable in performance if it has high mean performance, unit regression coefficient, and least deviation from regression. Cultivar with a regression 
value above one is considered unstable with higher sensitivity to environment change. It is good for specific adaptation in high yielding environment. Regression coefficient below one indicates that the cultivar is relatively stable with greater resistance to environment change. KKU-P-21041 was the most stable for fruit yield with above average fruit yields. This cultivar performed uniformly in all environments. Earlier studies by Zewdie and Poulos (1996) on KKU-P-21041 (Punjab Lal) assessed across seven environments of different countries showed that Punjab Lal was stable but with low yield. Although direct comparisons of results are considered irrelevant as the environments are different (Pritts and Luby 1990), our results are comparable on stability. Therefore, KKU-P-21041 is a good cultivar on yield stability. KKU-P-11015 and Dallay khorsaney is good for specific locations as the yield was high and was highly responsive to favorable environments. However, Dallay khorsaney was sensitive to dry season environments.

Considering our results on capsaicinoids, three groups of stability was observed for capsaicinoids which corresponds to the pungency level. KKU-P11003 , a medium pungency cultivar with regression values almost equal to one and low deviation from regression performed consistently for fruit yield, individual, and total capsaicinoids across six environments. Therefore, this was the most stable cultivar for capsaicinoids and could be selected for stability of capsaicinoid production. High pungency cultivars; Dallay khorsaney, KKU-P-21041 and KKU-P-22006 showed regression values more than one in most traits and high deviation from regression indicated its adaptation to specific locations. The low pungency varieties; KKU-P-11015 and KKU-P-21041 were least responsive with low regression values indicating more stability although deviations from regression were high. Zewdie and Bosland (2000) also reported high stability in genotypes with low capsaicinoid content and low stability in high capsaicinoid content which corresponds to our results. The result also showed a general tendency that a relatively stable capsaicin contents corresponds to the stable total capsaicinoids. This could be due to higher capsaicin concentration than dihydrocapsaicin. The stability in total capsaicinoids did not mean stability in individual capsaicinoids and vice versa as was observed in KKU-P22006. This could be because of different gene actions and the inheritance studies reports that different genes are controlling the synthesis of each capsaicinoid (Garcés-Claver et al. 2007). However, because of the significant difference from unity and the large deviation from regression we could not conclude stability of other cultivars except KKU-P-11003 which was stable for all characters, across all locations. Since KKU-P-11003 showed good responses and was stable for stability for fruit yield, individual and total capsaicinoids across six environments, it could be a good genetic source for stability in breeding programs for capsaicinoids.

Acknowledgments The authors are grateful to the Thailand International Development Cooperation Agency, the National Science of Thailand and Development Association, Research and Technology Transfer Office and the Plant Breeding Research Center for Sustainable Agriculture, Khon Kaen University, for providing us the research funds. We also thank the Royal Project Foundation Research Centre, Pangda Chiang Mai Thailand, Royal University of Bhutan, the College of Natural Resources, the assistant agriculture extension officer and the farmers of Kabesa, Bhutan for their support during field experiments.

Open Access This article is distributed under the terms of the Creative Commons Attribution License which permits any use, distribution, and reproduction in any medium, provided the original author(s) and the source are credited.

\section{References}

Collins MD, Wasmund LM, Bosland PW (1995) Improved method for quantifying capsaicinoids in Capsicum using high-performance liquid chromatography. HortScience 30:137-139

Contreras-Padilla M, Yahia EM (1998) Changes in capsaicinoids during development, maturation and senescence of chile pepper and relation with peroxidase activity. J Agric Food Chem 46:2075-2079

Eberhart SA, Russell WA (1966) Stability parameters for comparing varieties. Crop Sci 6:36-40

Estrada B, Pomar F, Diaz J, Merino F, Bernal MA (1999) The effect of seasonal changes on the pungency level of Padron pepper fruit. Capsicum Eggplant Newsl 18:28-31

Finlay KW, Wilkinson GN (1963) The analysis of adaptation in plant breeding program. Aust J Agric Res 14:742-754

Freeman GH, Dowker BD (1973) Statistical methods for the analysis of genotype - environments. Heredity 33:339-354

Garcés-Claver A, Gil-Ortega R, Álvarez-Fernández A, ArnedoAndrés MS (2007) Inheritance of capsaicin and dihydrocapsaicin, determined by HPLC-ESI/MS, in an intraspecific cross of Capsicum annuum L. J Agric Food Chem 55: 6951-6957 
Gill KS, Nanda GS, Singh G (1984) Stability analysis over seasons and locations of multilines of wheat (Triticum aestivum L.). Euphytica 33:489-495

Gomez KA, Gomez AA (1984) Statistical procedures for agricultural research, 2nd edn. Wiley, New York

Gurung T, Techawongstien S, Suriharn B, Techawongstien S (2011) Impact of environments on the accumulation of Capsaicinoids in Capsicum spp. HortScience 46(12):15761581

Harvell KP, Bosland PW (1997) The environment produces a significant effect on pungency of chiles. HortScience 32:1292

Iwai K, Suzuki T, Fujiwake H (1979) Formation and accumulation of pungent principle of hot pepper fruits, capsaicin and its analogues, in Capsicum annuиm var. annuиm cv. Karayatsubusa at different growth stages after flowering. Agric Biol Chem 43:2493-2498
Pritts M, Luby J (1990) Stability indices for horticulture crops. HortScience 25:740-745

Rasamivelona A, Gravois KA, Dilday RH (1995) Heritability and genotype $\times$ environment interactions for strait head in rice. Crop Sci 35:1365-1368

Russel OF (1994) MSTAT-C v.2.1 (a computer based data analysis software). Crop and Soil Science Department, Michigan State University, USA

Zewdie Y, Bosland PW (2000) Evaluation of genotype, environment, and genotype-by-environment interaction for capsaicinoids in Capsicum annuum L. Euphytica 111:185-190

Zewdie Y, Poulos JM (1996) Stability analysis in hot pepper. Capsicum Eggplant Newsl 14:39-42 